\title{
MISSÃO ORGANIZACIONAL: ANÁLISE DOS PRINCIPAIS ELEMENTOS PRESENTES NAS MISSÕES DAS EMPRESAS HOTELEIRAS BRASILEIRAS
}

ORGANIZATIONAL MISSION: ANALYSIS OF THE MAIN ELEMENTS PRESENT IN THE MISSION OF BRAZILIAN HOTEL COMPANIES

MISIÓN ORGANIZACIONAL: ANÁLISIS DE LOS PRINCIPALES ELEMENTOS PRESENTES EN LAS MISIONES DE LAS EMPRESAS HOTELERAS BRASILEÑAS

\section{Jaqueline Freitas Vilain}

Graduanda em Ciências Contábeis pela Universidade Federal de Santa Catarina jaquelinevilain@hotmail.com

\section{Paula de Souza}

Doutoranda em Contabilidade pela Universidade Federal de Santa Catarina Mestre em Contabilidade pela Universidade Federal de Santa Catarina pauladesouza1604@gmail.com

\section{Rogério João Lunkes}

Pós-doutor em Contabilidade pela Universitat de València Professor da Universidade Federal de Santa Catarina rogerio.lunkes@ufsc.br

Data de Submissão: 15/03/2015 Data de Aprovação: 22/09/2015

Resumo: A missão organizacional é a principal proposta da empresa, pois demonstra para que ela serve, a justificativa de sua existência para a sociedade e a função social que ela exerce. A declaração de missão representa uma potencial ferramenta para a formulação e a implementação 
da estratégia de uma organização. Nesse contexto, este artigo tem por objetivo identificar e analisar os principais elementos presentes nas missões das empresas hoteleiras brasileiras associadas à Associação Brasileira da Indústria de Hotéis (ABIH). Efetuou-se um estudo de cunho descritivo, com abordagem quantitativa e análise de conteúdo. A relação dos hotéis investigados foi extraída do website da $\mathrm{ABIH}$, totalizando 2056 empresas hoteleiras. A amostra foi composta por 243 hotéis que divulgaram nele sua missão. Estabeleceu-se o modelo de Pearce II (1982) como indexador de missão efetiva. Os resultados indicam que nenhuma empresa da amostra possui uma missão efetiva, isto é, os hotéis brasileiros fazem a missão por adaptação à sua estrutura ou influência do setor em que estão inseridos. Os indicativos apontam que as empresas pesquisadas utilizam a missão mais como instrumento de propaganda do que de gestão estratégica.

Palavras-chave: Missão organizacional. Elementos. Hotéis.

Abstract: The organizational mission is the main purpose of the company, what it is for, the justification for its existence to society, and the social role it plays. The mission statement is a potential tool for formulating and implementing the strategy of an organization. In this context, this article aims to identify and analyze the main elements present in the missions of Brazilian hotel companies that are members of the Brazilian Hotel Industry Association (ABIH). We conducted a descriptive study, using a quantitative approach and content analysis. The list of hotels investigated was taken from the ABIH website, totaling 2056 hotel companies. The sample consisted of 243 hotels that have reported their mission. The Pearce II model (1982) was established as an effective mission indexer. The results indicate that no company in the sample has an effective mission, i.e. the mission of Brazilian hotels consists of adapting their structure or influencing the sector in which they are inserted. The indications show that the companies surveyed use the mission statement more as a propaganda tool than as a form of strategic management.

Keywords: Organizational mission. Elements. Hotels.

Resumen: La misión organizacional es la principal propuesta de la empresa, pues demuestra para qué sirve, la justificación de su existencia para la sociedad y la función social que ejerce. La declaración de la misión representa una potencial herramienta para la formulación y la implementación de la estrategia de una organización. En ese contexto, este artículo tiene por objetivo identificar y analizar los principales elementos presentes en las misiones de las empresas hoteleras brasileñas que forman parte de la Asociación Brasileña de la Industria de Hoteles (ABIH). Se efectuó un estudio de cuño descriptivo, con un abordaje cuantitativo y análisis de contenido. La lista de los hoteles investigados fue extraída del website de la $\mathrm{ABIH}$, totalizando 2056 empresas hoteleras. La muestra estaba compuesta por 243 hoteles que divulgaron su misión en el mismo. Se estableció el modelo de Pearce II (1982) como indexador de misión efectiva. Los resultados indican que ninguna de las empresas de la muestra posee una misión efectiva, es decir que los hoteles brasileños hacen la misión por adaptación a su estructura o influencia del sector al que pertenecen. Los indicativos señalan que las empresas estudiadas utilizan la misión más como instrumento de propaganda que de gestión estratégica.

Palabras clave: Misión organizacional. Elementos. Hoteles. 


\section{INTRODUÇÃO}

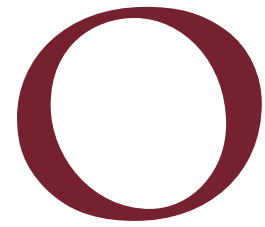

turismo brasileiro evoluiu consideravelmente nos últimos anos.

De acordo com o Conselho Mundial de Viagens e Turismo, os números do Brasil mostram que o setor apresentou uma contribuição total - que compreende as atividades diretas, indiretas e induzidas do turismo - de 9,2\% do Produto Interno Bruto (PIB). No que diz respeito à contribuição direta do setor no país, o percentual é de 3,5\% do PIB (MINISTÉRIO DO TURISMO, 2014).

Quanto à expectativa para o setor hoteleiro, existe a necessidade de se preparar para receber os turistas e conceder hospedagem de qualidade, proporcionando serviços de valor agregado. Porém, tudo isso exige gestão interna eficiente do setor hoteleiro (ARAÚJO; SOUZA; LUNKES, 2014).

A diversidade do mercado aliada à evolução tecnológica eà forte concorrência faz com que as empresas hoteleiras precisem de melhor gerenciamento e controle de suas atividades. Por isso, carecem cada vez mais de especialização e melhoria da gestão, buscando o diferencial no ambiente competitivo.

Nesse sentido, a utilização do planejamento estratégico surge com o propósito de auxiliar a gestão hoteleira empresarial. Para Rocha, Miguel e Oliveira Júnior (2012), o planejamento estratégico é uma ferramenta importante, que permite as empresas traçarem seus objetivos de crescimento e caminhos para alcançá-los, além de prever a relação entre as empresas e o ambiente interno e externo.

Uma declaração de missão é uma potencial ferramenta para a formulação e a implementação da estratégia de uma organização (BAETZ; BART, 1996). Ademais, a missão tem refletido o processo de planejamento estratégico em um leque relativamente amplo de organizações, incluindo as sem fins lucrativos (BUSCH; FOLARON, 2005).

A missão é a principal proposta da empresa, para que ela serve, a justificativa de sua existência para a sociedade e a função social que ela exerce (TAKESKI; REZENDE, 2000). Isto implica dizer que ela estabelece os objetivos, formula as estratégias, as metas e as políticas, combinando destinação de recursos à motivação dos 
funcionários. A missão organizacional é, à vista disso, um componente integral do processo de gestão estratégica (COCHRAN; DAVID; GIBSON, 2008).

Devido ao exposto, Pearce II (1982) propôs uma estrutura com oito elementos que devem estar compreendidos na frase da missão organizacional, de modo a torná-la uma ferramenta efetiva de planejamento estratégico. Diante disso, tem-se a pergunta de pesquisa que direciona este artigo: quais são os principais elementos presentes nas missões das empresas hoteleiras brasileiras? A fim de responder a questão-problema, o objetivo deste estudo é identificar e analisar os principais elementos presentes nas missões das empresas hoteleiras brasileiras associadas à Associação Brasileira da Indústria de Hotéis (ABIH).

Este estud o contribui para o desenvolvimento das missões organizacionais em empresas do setor hoteleiro, posto que são fundamentais para a sobrevivência, o crescimento e o sucesso de qualquer negócio (ANALOUI; KARAMI, 2002; BARTKUS; GLASSMAN; MCAFEE, 2006; WILLIAMS, 2008).

\section{FUNDAMENTAÇÃO TEÓRICA}

\section{CONCEITO DE MISSÃO}

Há mais de 30 anos a missão serve como uma ferramenta usual de comunicação corporativa (WILLIAMS, 2008). Descreve e comunica a finalidade primordial de todas as partes interessadas de uma organização (KEMP; DWYER, 2003).

Crença, filosofia, valores fundamentais, criação de imagem ou um fator distintivo são conceitos frequentemente utilizados para delinear a importância e o valor de uma declaração de missão para uma empresa (DERMOL; BREZNIK, 2012).

Pesquisadores sustentam que a missão representa essencialmente a "razão de ser" das empresas (DRUCKER, 1974; TAKESKI; REZENDE, 2000; ALMEIDA, 2003; RAFAELI; CAMPAGNOLO; MÜLLER, 2007; COCHRAN; DAVID; GIBSON, 2008; WILLIAMS, 2008; DAVID, 2009; LOBATO, 2009; MCKEE, 2012). 
De acordo com David (2009), a razão de existir da organização deve ser claramente indicada na missão, transmitindo informações importantes, tais como: os produtos e os serviços oferecidos, os principais mercados, a preocupação da empresa com o crescimento, a eficiência, os funcionários, os acionistas, o meio ambiente e a rentabilidade em longo prazo.

Consequentemente, uma declaração de missão que expressa a razão da organização, transmite a existência, a identidade, o foco e a direção para os seus stakeholders (GEMMEL, 2002).

A declaração de missão é um documento formal da visão que expressa o que a empresa quer ser e o que deveria ser. Todas as empresas têm uma missão, mesmo que não seja por escrito. Contudo, o processo de desenvolver uma declaração escrita necessita que a gestão contemple, conscientemente, a natureza do negócio, os clientes e os mercados, o que serve e deve ser servido, bem como os produtos/serviços que estão e devem ser comercializados (DRUCKER, 1974; PEARCE II, 1982).

Para Matejka, Kurke e Gregory (1993), a missão pinta o presente para o futuro. A partir dela, a organização gera metas adequadas (metas/objetivos). Essas metas se tornam barômetros ao longo do caminho, ajudando a organização a se mover em direção à visão e fornecendo pontos de verificação para avaliar o seu progresso.

Uma declaração de missão bem trabalhada pode servir como um mecanismo de controle, como forma de concentrar a gestão na tomada de decisão para criar um equilíbrio entre os interesses conflitantes de várias partes interessadas e para motivar e inspirar os demais membros da organização (DESMIDT; PRINZIE; HEENE, 2008).

Por outro lado, a obscuridade da missão organizacional, segundo Rivera (1992), gera impossibilidade de definição das funções internas, o que fragiliza a capacidade de gestão da administração.

Considerando a missão a razão de ser de uma organização, constata-se que é necessário às empresas que desejam permanecer competitivas no mercado atuarem estrategicamente e terem a missão como orientadora de seus negócios. 


\section{FINALIDADES E BENEFÍCIOS DA MISSÃO}

Uma quantidade crescente de literatura (CAMPBELL; YEUNG, 1991; BART; BONTIS; TAGGAR, 2001) aponta a declaração de missão como uma ferramenta valiosa para melhorar o desempenho organizacional e aumentar o nível de satisfação e motivação dos colaboradores (CAMPBELL; YEUNG, 1991; KLEMM; SANDERSON; LUFFMAN, 1991; COLLINS; PORRAS, 1996; LENCIONI, 2002).

A missão consiste em normas e valores fortes que influenciam a maneira pela qual as pessoas se comportam. Ou seja, seus componentes refletem o nível de consenso que existe entre os membros da organização e o seu propósito (LIN, 2012).

Os componentes da missão devem motivar e inspirar os funcionários a alcançarem um objetivo ou propósito comum (KLEMM; SANDERSON; LUFFMAN, 1991); equilibrar os interesses conflitantes de diferentes stakeholders (KLEMM; SANDERSON; LUFFMAN, 1991); criar padrões de comportamento (BART, 1996); fornecer um objetivo e direção comuns (CAMPBELL; YEUNG, 1991); definir o âmbito das atividades (CAMPBELL; YEUNG, 1991); permitir o controle sobre a organização (KLEMM; SANDERSON; LUFFMAN, 1991); e desenvolver valores e cultura compartilhados dentro da organização (CAMPBELL; YEUNG, 1991).

As declarações de missão que incluem componentes específicos, ou seja, competência distintiva, mercado-alvo, produtos/serviços, identidade única, imagem pública desejada, preocupação com a satisfação dos clientes e preocupação com os funcionários estão associadas com o desempenho financeiro (BARTKUS; GLASSMAN; MCAFEE, 2006).

Lobato (2009) acrescenta que a missão deve ser sintética e compreensível, além de incluir aspectos norteadores das definições estratégicas da empresa: qual o negócio; quem é seu cliente; qual o seu escopo; qual a sua vantagem competitiva; qual a sua contribuição social; e quais são os seus valores.

A empresa que almeja ter continuidade deve saber precisamente quem é, onde está e qual lugar quer alcançar. A definição da missão organizacional responde a essas perguntas, além de possibilitar trazer diversos benefícios para a entidade. 
Vasconcellos Filho e Pagnoncelli (2001) defendem que a missão proporciona alguns benefícios essenciais para o sucesso de uma organização: orienta a partida; evita a armadilha do sucesso; funciona como farol alto; atrai, motiva e retém talentos; orienta a formulação dos objetivos; e ajuda a aumentar a produtividade.

Ressalta-se que é de extrema importância agir de forma cautelosa ao desenvolver ou elaborar a missão organizacional, considerando-se seus potenciais benefícios e seu papel fundamental nos processos de avaliação de desempenho (VANDIJCK; DESMIDT; BUELENS, 2007).

Nesse contexto, Pearce II (1982) elaborou um modelo com oito principais elementos para compor uma missão organizacional, a qual deve conter: principais clientes e mercados; preocupação com a imagem pública; sobrevivência, crescimento e lucratividade; valores e filosofia corporativa; produtos ou serviços; competência própria/distintiva; domínio geográfico; e tecnologia.

\section{ESTUDOS ANTERIORES}

Diversos estudos foram desenvolvidos acerca da temática de missão organizacional. Na década de 70, Drucker (1974) já defendia que as missões deveriam conter ao menos três elementos básicos, como: produtos ou negócio; mercados ou seus consumidores e postura estratégica adotada. Anos depois, Want (1986) indicou a abrangência de cinco elementos, quais foram: razão da existência; metas do negócio; identidade da organização; políticas; e seus valores e filosofias.

Posteriormente, Rarick e Vitton (1995) estudaram os elementos presentes nas missões de grandes empresas consideradas de sucesso nos Estados Unidos. Já a pesquisa de Bart e Baetz (1998) analisou a relação entre as missões organizacionais e o desempenho de 136 grandes empresas do Canadá.

O'Gorman e Doran (1999) averiguaram a existência da relação entre as missões organizacionais abrangentes e o elevado crescimento de pequenas e médias empresas irlandesas. Enquanto isso, Smith et al. (2001) realizaram uma pesquisa em que avaliaram a relação entre os conteúdos presentes nas missões e a longevidade organizacional. 
Omran, Atrill e Pointon (2002) investigaram se as empresas que adotam uma abordagem de partes interessadas e demonstram competência mais ampla de responsabilidade corporativa proporcionam retornos menores aos que adotam a abordagem de valor para os acionistas.

De outro modo, Anloui e Karami (2002) examinaram as percepções dos CEOs em relação às missões organizacionais de 508 pequenas e médias empresas do setor elétrico e eletrônico do Reino Unido.

O estudo de Biloslavo (2004) averiguou as missões organizacionais divulgadas nos sites das 50 maiores empresas da Eslovênia. Ao mesmo tempo, Toftoy e Chatterjee (2004) estudaram as missões de pequenas empresas.

Bartkus, Glassman e McAfee (2006) observaram a relação entre a qualidade das missões organizacionais e o desempenho financeiro de 30 empresas do Japão, da Europa e dos Estados Unidos.

Van Nimwegen et al. (2008) objetivaram alcançar evidências para a relação teórica entre a importância dos stakeholders para a empresa e a inclusão dos grupos de interessados nas missões das empresas, utilizando 490 observações. Bartkus e Glassmann (2008) pesquisaram a relação entre o teor das missões e as ações de gerenciamento dos stakeholders, ou seja, se as organizações de fato praticam o que está definido em suas missões.

Ainda que os estudos internacionais sobre as missões tenham alcançado propósitos distintos, existem alguns deles que testaram ou empregaram a proposta de missão efetiva de Pearce II (1982) em diversas organizações (PEARCE; DAVID, 1987; DAVID, 1989; MORRIS, 1996; KEMP; DWYER, 2003; PEYREFITTE; DAVID, 2006; RARICK; NICKERSON, 2006; PALMER; SHORT, 2008; WILLIAMS, 2008).

No Brasil foram realizadas quatro principais pesquisas que empregaram os oito elementos de Pearce II (1982) (AQUINO, 2003; MUSSOI; LUNKES; SILVA, 2011; TELES; LUNKES, 2011; ARAÚJO; SOUZA; LUNKES, 2014).

A Tabela 1 apresenta, em ordem cronológica, os resultados encontrados nos trabalhos internacionais e nacionais que utilizaram os oito elementos propostos por Pearce II (1982). 
Tabela 1. Resultados dos trabalhos que utilizaram os elementos de Pearce II (1982)

\begin{tabular}{|c|c|c|c|c|}
\hline Autores & Pearce e David & David & Morris & Kemp e Dwyer \\
\hline Ano & 1987 & 1989 & 1996 & 2003 \\
\hline País/Amostra & \multirow{2}{*}{$\begin{array}{l}\text { EUA/500 } \\
\text { Fortune }\end{array}$} & \multirow{2}{*}{$\begin{array}{l}\text { EUA/500 } \\
\text { Fortune }\end{array}$} & \multirow{2}{*}{$\begin{array}{l}\text { EUA/500 } \\
\text { Fortune }\end{array}$} & \multirow{2}{*}{$\begin{array}{l}\text { Mundial/ Cias } \\
\text { Aéreas }\end{array}$} \\
\hline $\begin{array}{l}\text { Frequência de elementos } \\
\text { encontrados }\end{array}$ & & & & \\
\hline Principais clientes e mercados & $54 \%$ & $83 \%$ & $42 \%$ & $72 \%$ \\
\hline Produtos ou serviços & $72 \%$ & $80 \%$ & $56 \%$ & $32 \%$ \\
\hline Domínio geográfico & $38 \%$ & - & $54 \%$ & $42 \%$ \\
\hline Tecnologia & $11 \%$ & $30 \%$ & $15 \%$ & $22 \%$ \\
\hline $\begin{array}{l}\text { Sobrevivência, crescimento ou } \\
\text { lucrativa }\end{array}$ & $91 \%$ & $86 \%$ & $48 \%$ & $48 \%$ \\
\hline Valores e filosofia corporativa & $65 \%$ & $25 \%$ & $44 \%$ & $80 \%$ \\
\hline Competência própria/distintiva & $71 \%$ & $18 \%$ & $67 \%$ & $88 \%$ \\
\hline Preocupação com a imagem pública & $87 \%$ & $75 \%$ & $24 \%$ & $30 \%$ \\
\hline $\begin{array}{l}\text { Autores } \\
\text { Ano } \\
\text { País/Amostra }\end{array}$ & \multirow[t]{2}{*}{$\begin{array}{c}\text { Peyrefitte e } \\
\text { David } \\
2006 \\
\text { EUA/Maiores }\end{array}$} & \multirow{2}{*}{$\begin{array}{c}\text { Rarick e } \\
\text { Nickerson } \\
2006 \\
\\
\text { EUA/1000 } \\
\text { BW }\end{array}$} & \multirow{2}{*}{$\begin{array}{c}\text { Palmer e } \\
\text { Short } \\
2008 \\
\\
\text { EUA/ } \\
\text { Escolas }\end{array}$} & \multirow{2}{*}{$\begin{array}{l}\text { Williams } \\
2008 \\
\text { EUA/1000 } \\
\text { Fortune }\end{array}$} \\
\hline $\begin{array}{l}\text { Frequência de elementos } \\
\text { encontrados }\end{array}$ & & & & \\
\hline Principais clientes e mercados & $74 \%$ & $40 \%$ & $49 \%$ & $86 \%$ \\
\hline Produtos ou serviços & $86 \%$ & $96 \%$ & $94 \%$ & $70 \%$ \\
\hline Domínio geográfico & $24 \%$ & $13 \%$ & $50 \%$ & $71 \%$ \\
\hline Tecnologia & $35 \%$ & $24 \%$ & $10 \%$ & $26 \%$ \\
\hline $\begin{array}{l}\text { Sobrevivência, crescimento ou } \\
\text { lucrativa }\end{array}$ & $60 \%$ & $51 \%$ & $33 \%$ & $58 \%$ \\
\hline Valores e filosofia corporativa & $37 \%$ & $47 \%$ & $36 \%$ & $53 \%$ \\
\hline Competência própria/distintiva & $70 \%$ & $60 \%$ & $72 \%$ & $63 \%$ \\
\hline Preocupação com a imagem pública & $33 \%$ & $47 \%$ & $62 \%$ & $60 \%$ \\
\hline $\begin{array}{l}\text { Autores } \\
\text { Ano } \\
\text { País/Amostra }\end{array}$ & \multirow{2}{*}{$\begin{array}{l}\text { Aquino } \\
2003 \\
\text { Brasil } \\
17 \text { IES }\end{array}$} & \multirow{2}{*}{$\begin{array}{l}\text { Mussoi et al } \\
2011 \\
\text { Brasil } \\
140 \text { Bovespa }\end{array}$} & \multirow{2}{*}{$\begin{array}{c}\text { Teles e } \\
\text { Lunkes } \\
2011 \\
\text { Brasil } \\
\text { Exame } \\
2009 \\
\end{array}$} & \multirow{2}{*}{$\begin{array}{l}\text { Araújo, Souza e } \\
\text { Lunkes } \\
2014 \\
\text { Universidades }\end{array}$} \\
\hline $\begin{array}{l}\text { Frequência de elementos } \\
\text { encontrados }\end{array}$ & & & & \\
\hline Principais clientes e mercados & $88 \%$ & $96 \%$ & $83 \%$ & - \\
\hline Produtos ou serviços & - & $44 \%$ & $52 \%$ & $23 \%$ \\
\hline Domínio geográfico & $29 \%$ & $15 \%$ & $28 \%$ & - \\
\hline Tecnologia & $41 \%$ & $7 \%$ & $5 \%$ & $31 \%$ \\
\hline $\begin{array}{l}\text { Sobrevivência, crescimento ou } \\
\text { lucrativa }\end{array}$ & - & $58 \%$ & $45 \%$ & $81 \%$ \\
\hline Valores e filosofia corporativa & $53 \%$ & $56 \%$ & $37 \%$ & $20 \%$ \\
\hline Competência própria/distintiva & - & $30 \%$ & $87 \%$ & $33 \%$ \\
\hline Preocupação com a imagem pública & - & $94 \%$ & $90 \%$ & $23 \%$ \\
\hline
\end{tabular}

Fonte: Adaptada de Mussoi, Lunkes e Silva (2011). 
Os elementos mais encotrados nas missões dos estudos anteriores foram "principais clientes e mercados" e "produtos ou serviços", e o elemento menos citad o foi "tecnologia". Esta carência de estudos anteriores envolvendo os elementos da moldura de Pearce II (1982) manifesta a importância de se realizar também pesquisa acerca das missões organizacionais em empresas do setor hoteleiro.

\section{ASPECTOS METODOLÓGICOS}

\section{ENQUADRAMENTO METODOLÓGICO}

O enquadramento metodológico da presente pesquisa está dividido em: natureza do objetivo, procedimentos da pesquisa e abordagem do problema. Quanto aos objetivos é considerada descritiva, uma vez que tem a finalidade de descrever e interpretar os elementos propostos por Pearce II (1982) contidos nas missões dos hotéis brasileiros (GIL, 2010).

Em relação aos procedimentos, a técnica utilizada é a análise de conteúdo, tendo em vista que são empregados métodos para estudar a descrição dos elementos de Pearce II (1982), possibilitando fazer inferências relativas às missões organizacionais. Para Flick (2009), a análise de conteúdo é um dos procedimentos para analisar o material textual, independente da origem desse material.

No que diz respeito à abordagem do problema, a pesquisa é caracterizada como quantitativa. De acordo com Diehl e Tatim (2004), define-se pesquisa quantitativa como o uso da qualificação tanto na coleta quanto no tratamento das informações, visando garantir resultados e evitar distorções de análise e interpretação.

\section{POPULAÇÃO E AMOSTRA}

A população desta pesquisa é constituída particularmente por empresas hoteleiras do Brasil que estão associadas à Associação Brasileira da Indústria de Hotéis $(A B I H)$ e que estavam listadas no website da referida Associação no período de 17 de setembro de 2014 a 10 de fevereiro de 2015. 
A seleção por hotéis e pousadas brasileiras associadas se justifica pela facilidade de acesso aos seus sítios eletrônicos, os quais estão todos disponíveis de modo gratuito no website da ABIH.

O Brasil é um país composto por 27 unidades federativas, sendo destas 26 estados e um distrito federal. De modo a facilitar a análise dos dados, os estados foram dividos nas regiões Norte, Nordeste, Centro-Oeste, Sudeste e Sul. A Tabela 2 evidencia o quantitativo dos hotéis por região.

Tabela 2. Quantitativo dos hotéis por região

\begin{tabular}{|c|c|c|c|c|c|c|c|}
\hline Região & Hotéis & $\begin{array}{c}\text { Membro } \\
\text { de rede }\end{array}$ & $\%$ & $\begin{array}{l}\text { Sem } \\
\text { site }\end{array}$ & $\%$ & $\begin{array}{c}\text { Divulga missão } \\
\text { em website* }\end{array}$ & $\%$ \\
\hline Norte & 59 & 5 & 11,80 & 12 & 20,34 & 8 & 13,56 \\
\hline Nordeste & 1117 & 112 & 10,03 & 117 & 10,47 & 120 & 10,74 \\
\hline Centro-Oeste & 166 & 23 & 13,86 & 21 & 12,65 & 34 & 20,48 \\
\hline Sudeste & 497 & 150 & 30,18 & 14 & 2,81 & 61 & 12,27 \\
\hline Sul & 217 & 17 & 7,83 & 18 & 8,29 & 20 & 9,21 \\
\hline
\end{tabular}

*Excluídas as missões duplicadas dos hotéis membros de rede.

Fonte: Dados da pesquisa.

A coleta das missões organizacionais foi feita por meio dos sítios eletrônicos dos próprios hotéis. Primeiramente foram selecionadas 2056 empresas. Deste total, 182 não possuíam ou tinham indisponíveis seus websites e 307 eram membros de redes e, portanto, apresentavam a mesma missão. Sendo assim, a população se restringiu a 1567 hotéis. Constatou-se que, deste total, 243 hotéis possuíam missão definida e disponível no site, constituindo assim a amostra deste estudo.

Cabe ressaltar que $67,77 \%$ dos hotéis da Região Nordeste representam os hotéis associados do Estado da Bahia. Também, que a ABIH dos estados do Acre, Amapá, Pará, Roraima, Tocantins, Piauí, Mato Grosso e Distrito Federal não disponibilizaram a relação dos hotéis em seus sítios eletrônicos. 


\section{PROCEDIMENTOS PARA ANÁLISE DOS DADOS}

Para atingir a finalidade desta pesquisa, a análise dos dados foi dividida em dois momentos. No primeiro momento foram lidas as missões das empresas hoteleiras e identificados, com base em estudos anteriores, alguns dos elementos presentes nas missões. Tais elementos foram classificados conforme Ribeiro et al. (2008).

No segundo momento, os elementos encontrados foram comparados com a classificação proposta por Pearce II (1982). Consoante esse autor, para contribuir no processo do planejamento estratégico e ser eficaz, uma missão deve incluir alguns elementos básicos, que possibilitem a mensuração e a avaliação da estratégia adotada pela empresa. Optou-se pela forma de checklist para a indexação das informações, assim como na Tabela 1.

As limitações do estudo são presentes na extensão da amostra, tendo em vista que nem todas as empresas disponibilizaram as missões em seus websites e, também, no aspecto subjetivo da classificação dos enunciados em elementos.

RESULTADOS

O primeiro passo para a análise da efetividade da missão dos hotéis e das pousadas do Brasil, conforme modelo proposto por Pearce II (1982), é identificar quais os elementos as compõem. A Tabela 3 evidencia os principais elementos identificados de acordo com a pesquisa de Ribeiro et al. (2008).

Tabela 3. Frequência dos elementos encontrados nas missões dos hotéis brasileiros

\begin{tabular}{|c|c|c|c|c|c|c|}
\hline ELEMENTOS ENCONTRADOS NAS MISSÕES & 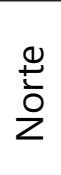 & 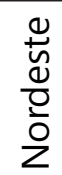 & 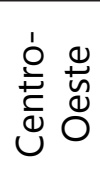 & $\overline{\bar{n}}$ & $\begin{array}{l}\stackrel{0}{ \pm} \\
\stackrel{0}{0} \\
\frac{0}{2} \\
\sim\end{array}$ & Brasil \\
\hline 1. Ênfase nos clientes & 6 & 80 & 28 & 39 & 39 & 192 \\
\hline 2. Produtos e Serviços & 3 & 63 & 16 & 33 & 40 & 155 \\
\hline 3. Contexto, setor econômico ou mercado de atuação & 5 & 25 & 5 & 7 & 19 & 61 \\
\hline 4. Preocupação com a qualidade & 6 & 62 & 19 & 24 & 34 & 145 \\
\hline 5. Retorno ao acionista & 0 & 12 & 7 & 11 & 9 & 39 \\
\hline 6. Filosofia e valores corporativos & 1 & 5 & 2 & 11 & 3 & 22 \\
\hline 7. Menção aos colaboradores & 0 & 31 & 17 & 10 & 18 & 76 \\
\hline 8. Responsabilidade Social & 0 & 13 & 7 & 9 & 4 & 33 \\
\hline 9. Conceito próprio/distintivo da empresa & 3 & 37 & 12 & 17 & 15 & 84 \\
\hline
\end{tabular}




\begin{tabular}{c|c|c|c|c|c|c}
\hline 10. Rentabilidade/Crescimento/ Sobrevivência & 0 & 20 & 10 & 20 & 6 & 56 \\
\hline $\begin{array}{c}\text { 11. Compromisso com o desenvolvimento (Sustentável/ } \\
\text { Econômico/Social) }\end{array}$ & 0 & 10 & 3 & 7 & 4 & 24 \\
\hline 12. Responsabilidade Ambiental & 1 & 8 & 1 & 3 & 1 & 14 \\
\hline 13. Local de atuação & 3 & 19 & 4 & 0 & 10 & 36 \\
\hline 14. Menção aos fornecedores & 0 & 5 & 2 & 5 & 0 & 12 \\
\hline 15. Tecnologia utilizada & 0 & 1 & 0 & 1 & 2 & 4 \\
\hline 16. Compliance com normas e leis & 0 & 1 & 0 & 0 & 1 & 2 \\
\hline TOTAL & $\mathbf{2 8}$ & $\mathbf{3 9 2}$ & $\mathbf{1 3 3}$ & $\mathbf{1 9 7}$ & $\mathbf{2 0 5}$ & 955 \\
\hline
\end{tabular}

Fonte: Adaptada de Ribeiro et al. (2008).

Ao analisar as 243 empresas brasileiras, encontrou-se o total de 955 elementos contidos nas missões. A ênfase em clientes, produtos e serviços, e a preocupação com a qualidade foram o foco da maioria, representando, respectivamente, $79 \%$, $64 \%$ e $60 \%$ do total de hotéis. Dentre os elementos com menor frequência estão a menção aos fornecedores, à tecnologia utilizada e ao compliance com normas e leis, sendo que este foi citado por apenas duas empresas.

Da mesma forma, na pesquisa feita nos Estados Unidos, com 500 empresas da Fortune (WILLIAMS, 2008), o elemento mais presente nas missões foi ênfase nos clientes. No Brasil, em estudos feitos em Instituições de Ensino por Aquino (2003) e nas Maiores e Melhores por Mussoi, Lunkes e Silva (2011) predomina a ênfase nos principais clientes e mercados.

Tal constatação demonstra a atenção que as empresas hoteleiras do Brasil despendem com seus hóspedes e produtos e serviços. Além do mais, esse cuidado está aliado à preocupação que os hotéis têm em prestar seus serviços e oferecer seus produtos com qualidade.

De outro modo, visualiza-se o baixo interesse dado pelo setor hoteleiro brasileiroàresponsabilidadeambientaleaocompromissocomo desenvolvimento sustentável/econômico/social. Isso implica dizer que, ainda que se preocupem com a qualidade dos produtos e dos serviços, as empresas hoteleiras não a relacionam com um compromisso sustentável e responsabilidade social, diferente do que sugerem Smith et al. (2001).

Outro elemento representativo em pesquisas internacionais é a rentabilidade/ crescimento/lucratividade.Porém, apenas $23 \%$ dasempresashoteleirasconsideram 
este aspecto. Isso significa que as missões, geralmente, estão direcionadas mais para o público externo, uma vez que a preocupação com a sobrevivência está relacionada, especialmente, com acionistas, funcionários e administradores.

Adicionalmente, o estudo de Anloui e Karami (2002) enfatiza a importância da missão para o resultado da empresa, pois constatou que cada vez mais o desempenho das entidades está sendo positivamente associado ao desenvolvimento das missões.

O segundo passo do trabalho destina-se a classificar os elementos constantes nas missões conforme a moldura de Pearce II (1982). A Tabela 4 apresenta a quantidade dos itens analisados.

Tabela 4. Elementos presentes nas missões de acordo com Pearce II (1982)

\begin{tabular}{|c|c|c|c|c|c|c|}
\hline ELEMENTOS PARA UMA MISSÃO EFETIVA & 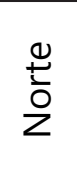 & 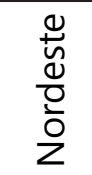 & 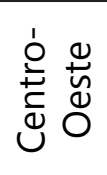 & $\overline{\bar{n}}$ & 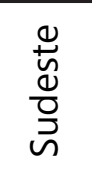 & Brasil \\
\hline 1. Principais clientes e mercados & 6 & 78 & 28 & 39 & 39 & 190 \\
\hline 2. Preocupação com a imagem pública & 2 & 62 & 20 & 3 & 35 & 122 \\
\hline 3. Sobrevivência, crescimento ou lucratividade & 1 & 18 & 10 & 20 & 9 & 58 \\
\hline 4. Valores e filosofia corporativa & 1 & 5 & 2 & 11 & 3 & 22 \\
\hline 5. Produtos ou serviços & 3 & 57 & 12 & 33 & 39 & 144 \\
\hline 6. Competência própria/distintiva & 3 & 37 & 13 & 17 & 15 & 85 \\
\hline 7. Domínio geográfico & 2 & 6 & 3 & 4 & 6 & 21 \\
\hline 8. Tecnologia & 0 & 1 & 0 & 1 & 2 & 4 \\
\hline TOTAL & 18 & 135 & 88 & 128 & 149 & 646 \\
\hline
\end{tabular}

Fonte: Dados da pesquisa.

Vislumbra-se, a partir da Tabela 4, que existem 646 elementos de Pearce II (1982), contraposto ao total de 955 de Ribeiro et al. (2008), presentes nas missões dos 243 hotéis analisados. Essa divergência se deve às diferentes metodologias empregadas pelas organizações e que resultou na ausência de alguns itens considerados indispensáveis para uma missão efetiva (PEARCE II, 1982).

O elemento mais frequente também foi principais clientes e mercados, com 190 (29\%) das aparições. Em segundo lugar estão os produtos ou serviços, que foi destacado por $22 \%$ do total dos hotéis. Entre os elementos menos presentes encontram-se a tecnologia $(0,62 \%)$, o domínio geográfico $(3,25 \%)$ e os valores e a filosofia corporativa $(3,40 \%)$. 
Concordando com esses achados, pesquisas realizadas em empresas internacionais (PEARCE; DAVID, 1987; MORRIS, 1996; KEMP; DWYER, 2003; PALMER; SHORT, 2008; WILLIAMS; 2008) e brasileiras (MUSSOI; LUNKES; SILVA, 2011; TELES; LUNKES, 2011) também apontam a tecnologia como o elemento menos citado nas frases das missões organizacionais.

Sob outro ponto de vista, os resultados se diferenciam dos estudos feitos por Want (1986), Rarick e Vitton (1995) e Bart e Baetz (1998), que apontaram a predominância dos valores, das crenças e da filosofia nas missões organizacionais das empresas.

No que concerne à efetividade da missão, notou-se que a maior parte das empresas possui entre 2 e 3 elementos. O maior número de elementos foi 5 , presentes em 13 empresas da amostra. Nenhuma empresa obteve 6, 7 e 8 elementos, implicando afirmar que não houve um hotel que contemplasse em sua missão os 8 elementos propostos por Pearce II (1982).

Nos estudos feitos por Mussoi, Lunkes e Silva (2011) e Teles e Lunkes (2011), as organizações também não empregaram a estrutura dos oito elementos de Pearce II (1982), mas algumas atingiram sete deles.

Os resultados demonstram que nenhuma empresa da amostra possui uma missão efetiva, avaliando a proposta de Pearce II (1982). Isso sugere que os hotéis brasileiros da amostra fazem a missão por adaptação à sua estrutura ou influência do setor em que estão inseridos. Desse modo, compreende-se que não é feito um exame dos elementos que compõem as missões, de maneira a gerar benefícios e torná-las um instrumento de planejamento estratégico.

Os indícios apontam que os hotéis pesquisados utilizam a missão organizacional mais como um instrumento de marketing e propaganda de seus produtos e serviços do que como uma ferramenta capaz de trazer melhorias para a gestão estratégica.

Por desfecho, para que a situação seja modificada, recomenda-se a conscientizaçãodosgestores/administradores/proprietáriossobrea importância de uma missão efetiva para o planejamento estratégico da empresa hoteleira. Tal ação auxiliará os gestores a identificar como tornar uma missão efetiva, além de orientá-los do impacto positivo que isso pode proporcionar para o hotel. 


\section{CONSIDERAÇÕES FINAIS}

O presente estudo analisou os elementos propostos por Pearce II (1982) presentes nas missões dos hotéis brasileiros. Foram identificadas e analisadas as missões de 243 hotéis associados à Associação Brasileira da Indústria de Hotéis (ABIH) que disponibilizaram as missões em seus respectivos websites.

No que diz respeito aos elementos de Ribeiro et al. (2008) evidenciados nas empresas hoteleiras, observou-se que o foco está nos clientes, nos produtos e nos serviços e na preocupação com a qualidade. Tais achados convergem com o de pesquisas anteriores (WILLIAMS, 2008; AQUINO, 2003; MUSSOI; LUNKES; SILVA, 2011).

Notou-se que os hotéis do Brasil analisados se preocupam com a qualidade dos produtos e dos serviços prestados. Contudo, de outro modo, dão baixa atenção ao compromisso com o desenvolvimento sustentável/econômico/social e à responsabilidade ambiental, ao contrário do que recomendam Smith et al. (2001).

Em relação aos oito elementos de Pearce II (1982), ressaltam-se principais mercados e clientes e produtos ou serviços. Diferentemente, Want (1986), Rarick e Vitton (1995), Bart e Baetz (1998) destacaram a presença de valores, crenças e filosofia corporativa nas missões organizacionais das empresas.

O elemento menos encontrado foi tecnologia, assim como em organizações de pesquisas anteriores (PEARCE; DAVID, 1987; MORRIS, 1996; KEMP; DWYER, 2003; PALMER; SHORT, 2008; WILLIAMS, 2008; MUSSOI; LUNKES; SILVA, 2011; TELES; LUNKES, 2011).

Os resultados também indicam que nenhuma empresa da amostra possui uma missão efetiva de Pearce II (1982), ou seja, as empresas hoteleiras brasileiras fazem a missão por adequação à sua estrutura ou a influência do setor em que estão inseridas. Mussoi, Lunkes e Silva (2011), Teles e Lunkes (2011) e Araújo, Souza e Lunkes (2014) também concluíram que as instituições não empregaram a missão efetiva.

Desse modo, a pesquisa aponta a necessidade da conscientização dos gestores sobre a relevância de uma missão efetiva para o planejamento estratégico da 
organização, uma vez que ela proporciona uma série de benefícios para o sucesso de uma empresa (VASCONCELLOS FILHO; PAGNONCELLI, 2001).

Em remate, recomenda-se a aplicação da pesquisa em empresas hoteleiras de outros países, outras áreas do setor como bares e restaurantes, além de outros setores da economia. Outrossim, sugere-se a realização de um estudo acerca do impacto das missões no desempenho das empresas e a percepção dos gestores quanto à importância desta ferramenta de gestão.

\section{REFERÊNCIAS}

ALMEIDA, M. I. R. Planejamento estratégico sendo aplicado em unidades de organizações e em profissionais. In: SEMEAD, 5., 2003, São Paulo. Anais... São Paulo, 2003.

ANLOUI, F.; KARAMI, A. CEOs and development of the meaningful mission statement. Corporate Governance, v. 2, n. 3, p. 13-20, 2002.

AQUINO, P. M. A construção e disseminação da missão nas Instituições de Ensino Superior do Ceará. In: ENCONTRO NACIONAL DOS PROGRAMAS DE PÓS-GRADUAÇÃO EM ADMINISTRAÇÂO, 27., 2003, Atibaia. Anais... Atibaia, 2003.

ARAÚJO, M. A.; SOUZA, P.; LUNKES, R. J. Análise dos principais elementos de Pearce II (1982) presentes nas missões organizacionais das universidades públicas do Brasil. In: COLÓQUIO INTERNACIONAL DE GESTÃO UNIVERSTIÁRIA, 41., 2014, Florianópolis. Anais... Florianópolis, 2014.

BAETZ, M. C.; BART, C. K. Developing mission statements which work. Long Range Planning, v. 29, n. 4, p. 526-533, 1996.

BART, C. K.; BAETZ, M. C. The relationship between mission statements and firm performance: an exploratory study. Journal of Management Studies, v. 35, n. 6, p. 823-853, 1998.

BART, C. K.; BONTIS, N.; TAGGAR, S. A model of the impact of mission statements on firm performance. Management Decision, v. 39, n. 1, p. 19-35, 2001.

BART, C. K. The impact of mission statements of firm innovativeness. International Journal of Technology Management, v. 11, n. 5, p. 479-493, 1996.

BARTKUS, B. R.; GLASSMAN, M. Do firms practice what they preach? The relationship between mission statements and stakeholder management. Journal Of Business Ethics, $v$. 83, n. 2, p. 207-216, 2008.

BARTKUS, B. R.; GLASSMAN, M.; MCAFEE, B. R. Mission statement quality and financial performance. European Management Journal, v. 24, n. 1, p. 86-94, 2006. 
BILOSLAVO, R. Web-based mission statements in Slovenian enterprises. Journal for East European Management, v. 9, n. 3, p. 265-278, 2004.

BUSCH, M.; FOLARON, G. Accessibility and clarity of state child welfare agency mission statements. Child Welfare, v. 84, p. 415-430, 2005.

CAMPBELL, A.; YEUNG, S. Creating a sense of mission. Long Range Planning, v. 24, n. 4, p. 10-20, 1991.

COCHRAN, D. S.; DAVID, F. R.; GIBSON, C. K. A framework for developing an effective mission statement. Journal of Business Strategies, v. 25, n. 2, p. 27-39, 2008.

COLLINS, J. C.; PORRAS, J. I. Building your company's vision. Harvard Business Review, v. 74, p. 65-77, 1996.

DAVID, F. R. How companies define their mission. Long Range Planning, v. 22, n. 1, p. 9097, 1989.

DAVID, F. R. Strategic management: concepts and cases. 20. ed. New York: Pearson Prentice Hall, 2009.

DERMOL, V.; BREZNIK, K. Identifying the mission statement's clusters in Slovenian Economy. Competences, Synergy and International Competitiveness Influence on the Technology Innovation and Industrial Management, 2012.

DESMIDT, S.; PRINZIE, A.; HEENE, A. The level and determinants of mission statement use: a questionnaire survey. International Journal of Nursing Studies, v. 5, n. 10, p. 1433-1441, 2008.

DIEHL, A. A.; TATIM, D. C. Pesquisa em ciências sociais aplicadas: métodos e técnicas. São Paulo: Prentice Hall, 2004.

DRUCKER, P. Management: tasks, responsibilities and practices. New York: Harper e Row, 1974.

FLICK, U. Introdução à pesquisa qualitativa. 3. ed. São Paulo: Artmed, 2009.

GEMMEL, P. Cursus Organisatiebeheer. Academiapress: Gent, 2002.

GIL, A. C. Como elaborar projetos de pesquisa. 5. ed. São Paulo: Atlas, 2010.

KEMP, S.; DWYER, L. Mission statements of international airlines: a content analysis. Tourism Management, v. 24, p. 635-653, 2003.

KLEMM, M.; SANDERSON, S.; LUFFMAN, G. Mission statements: selling corporate values to employees. Long Range Planning, v. 24, n. 3, p. 73-78, 1991. 
LENCIONI, P. M. Make your values mean something. Harvard Business Review, v. 80, n. 7, p. 113-117, 2002.

LIN, Y-H. Knowledge brokering for transference to the pilot's safety behavior. Management Decision, v. 50, n. 7, p. 1326-1338, 2012.

LOBATO, D. M. Estratégia de empresas. 9. ed. Rio de Janeiro: FGV, 2009.

MATEJKA, K.; KURKE, L. B.; BARB GREGORY. Mission Impossible? Designing a great mission statement to ignite your plans. Management Decision, v. 31, n. 4, p. 34-38, 1993.

MCKEE, A. Management: a focus on leaders. New York: Pearson Prentice Hall, 2012.

MINISTÉRIO DO TURISMO. Economia do turismo cresce no Brasil. 2014. Disponível em: http://www.turismo.gov.br/turismo/noticias/todas_noticias/2014041 7-1.html>. Acesso em: 02 fev. 2015.

MORRIS, R. J. Developing a mission for a diversified company. Long Range Planning, v. 29, ก. 1, 103-115, 1996.

MUSSOI, A.; LUNKES, R. J.; SILVA, R. V. Missão Institucional: uma análise da efetividade e dos principais elementos presentes nas missões de empresas brasileiras de capital aberto. Revista de Gestão, v. 18, n. 3, p. 361-384, 2011.

O'GORMAN, C.; DORAN, R. Mission statements in small and medium sized businesses. Journal of Small Business Management, v. 37, n. 4, p. 59-66, 1999.

OMRAN, M.; ATRILL, P.; POINTON, J. Shareholders versus stakeholders: Corporate mission statements and investor returns. Business Ethics: A European Review, v. 11, n. 34, p. 318327, 2002.

PALMER, T. B.; SHORT, J. C. Mission statements in U.S. colleges of business: an empirical examination of their content with linkages to configurations and performance. Academy of Management Learning and Education, v. 7, n. 4, p. 454-470, 2008.

PEARCE II, J. A. The company mission as strategic tool. Sloan Management Review, p. 1524, 1982.

PEARCE II, J. A.; DAVID, F. Corporate mission statement: the bottom line. The Academy of management Executive, v. 1, n. 2, p. 109-116, 1987.

PEYREFITTE, J.; DAVID, F. R. A content analysis of the mission Statements of united states firms in four industries. International Journal of Management, v. 23, n. 2, p. 296-301, 2006. 
RAFAELI, L.; CAMPAGNOLO, R. R. E MÜLLER, C. J. Missão declarada e missão desdobrada: uma abordagem para o planejamento estratégico. In: SIMPÓSIO DE ENGENHARIA DE PRODUÇÃO, 14., 2007, Bauru. Anais... Bauru, 2007.

RARICK, C, A.; NICKERSON, I. An empirical analysis of web-based corporate mission statements. Journal of Strategic E-commerce, v. 4, p. 1-2, 2006.

RARICK, C. A.; VITTON, J. Mission statement make cents. The Journal of Business Strategy, v. 16, n. 1, v. 11-12, 1995.

RIBEIRO, A. M.; SILVA, R. V.; LUNKES, R. J.; ALBERTON, L. Missão institucional: uma análise da efetividade e dos principais elementos presentes nas missões de empresas brasileiras de capital aberto. In: ENCONTRO NACIONAL DOS PROGRAMAS DE PÓS-GRADUAÇÃO EM ADMINISTRAÇÂO, 32., 2008, Rio de Janeiro. Anais... Rio de Janeiro, 2008.

RIVERA, U. Planejamento situacional: uma análise reconstrutiva. In: Gallo, E.; Rivera, U.; Machado, M. (Org.). Planejamento criativo: novos desafios em políticas de saúde. Rio de Janeiro: Relume Dumará; 1992.

ROCHA, D. P. M.; MIGUEL, J. H. S.; OLIVEIRA JÚNIOR, R. Planejamento estratégico nas micro e pequenas empresas: estudo quantitativo em empresas do ramo de materiais elétricos. Universitas, v. 5, n. 3, 2012.

SMITH, M.; HEADY, R. B.; CARSON, P. P.; CARSON, K. D. Do mission statements accomplish their missions? An exploratory analysis of mission statement content and organizational longevity. Journal of Applied Management and Entrepreneurship, v. 25, n. 6, p. 75-96, 2001.

TAKESKI, T.; REZENDE, W. Estratégia empresarial: tendências e desafios - um enfoque na realidade brasileira. São Paulo: Makron Books, 2000.

TELES, J.; LUNKES, R. J. Efetividade da missão institucional: um estudo nas 100 maiores empresas de capital aberto no Brasil. In: ANPCONT, 5., 2011, Vitória. Anais... Vitória, 2011.

TOFTOY, C. N.; CHATTERJEE, J. Mission Statements and the Small Business. Business Strategy Review, v. 15, p. 41-44, 2004.

VAN NIMWEGEN, G.; BOLLEN, L.; HASSINK, H.; THIJSSENS, T. A stakeholder perspective on mission statements: an international empirical study. International Journal of Organizational Analysis, v. 16, n. 1/2. p. 61-82, 2008.

VANDIJCK, D.; DESMIDT, S.; BUELENS, M. Relevance of mission statements in Flemish not-forprofit healthcare organizations. Journal of Nursing Management, v. 15, p. 131-141, 2007. 
ISSN: 1983-7151

VASCONCELLOS FILHO, P.; PAGNONCELLI, D. Construindo estratégias para vencer: um método prático, objetivo e testado para o sucesso da sua empresa. 12. ed. Rio de Janeiro: Campus, 2001.

WANT, J. H. Corporate mission. Management Review, v. 75, p. 46-50, 1986.

WILLIAMS, L. S. The mission statement: a corporate reporting tool with a past, present and future. Journal of business communication, v. 45, n. 2, p. 94-119, 2008. 